\title{
The Essential Role of Gold in the Fabrication of Microwave Electronics Systems
}

\author{
Giles Humpston \\ Marconi Materials Technology, Caswell, Towcester, Northamptonshire, NN12 8EQ
}

Received: 17 June 1999

Microwave technology will be the cornerstone of 21 st Century electronics. Microwave electronics systems differ radically from conventional silicon semiconductor technology, requiring specialist semiconductors, circuits and interconnects. Gallium arsenide and gallium nitride are advanced compound semiconductors that fulfil the first requirement. Gold and gold alloys have unique properties that are essential for integrating these components into functioning systems.

The second half of the 20th Century has been shaped by the explosive growth in electronics systems. Microchips can be found in almost every conceivable modem product, from toasters to computers and there is every indication that their application is set to expand further. The current drive in electronics technology is in communications systems. The World Wide Web and mobile telephones are obvious current examples, with automotive driver aids and interactive television on the technology horizon. Many of the proposed new applications require a radio or fibreoptic link for their function. The problem is that all of the easily accessible parts of the radio spectrum are already fully utilized so it is necessary to develop higher and higher frequency bands. The allocated automotive frequency band is $77 \mathrm{GHz}$ and local video systems could be in the $125 \mathrm{GHz}$ band.

Traditional silicon semiconductors are ideally suited to frequencies below about $5 \mathrm{GHz}$. Above this frequency compound semiconductors provide better functionality. Pre-eminent among these is gallium arsenide, which is likely to be superseded by gallium nitride for many applications requiring high power microwave circuits sometime in the future.

\section{MONOLITHIC MICROWAVE INTEGRATED CIRCUITS}

Microwave electronic circuits are fundamentally different from conventional devices. Whereas a state-of-the-art microprocessor will have up to 10 million transistors, its microwave counterpart will seldom contain more than 100 functional devices and often less than 10 if it is an analogue device. The reason for this difference is that at very high frequencies the size of standard wiring features is a significant fraction of the radio wavelength and hence they function as antennas. To compensate for the interaction that occurs between adjacent circuit elements requires exceedingly complex mathematical modelling tools and many hundreds of man-hours of design effort, giving minute attention to every aspect of the layout, are required for each circuit. Then, successful operation of the circuit is dependent on achieving the exacting physical tolerances in production.

For these reasons, the design, manufacture and utilization of microwave components is a highly specialized art. In order to facilitate their use, microwave chips are usually designed as units of functionality with simple interfaces, $e g$ an amplifier or mixer with nominally $50 \mathrm{ohm}$ input and output ports. These, very reproducible, monolithic microwave integrated circuits (MMICs) must be designed taking into account the substrate environment in which they are to be mounted, in order to obtain the correct performance. A change in the length of bond wire used, or in mounting method can cause mismatch at the interface ports, which can seriously degrade the circuit operation. 


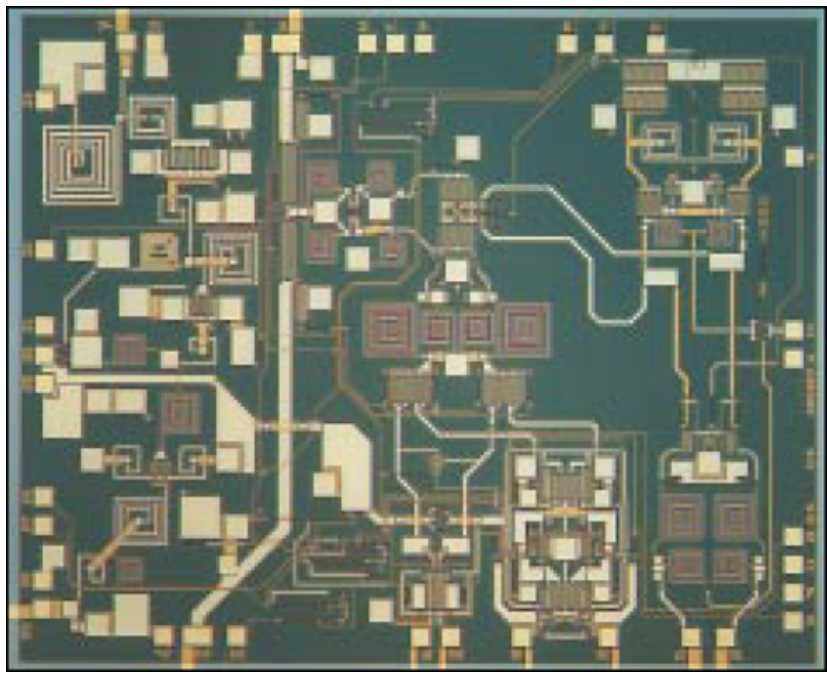

Figure 1 Monolithic Microwave Integrated Circuit This picture shows an MMIC of moderate complexity, diced from a wafer. The material is gallium arsenide. The active semiconductors are far too small to be seen. Most of the die area is occupied by spiral inductors, capacitor pads and interconnecting tracks. All of these are pure gold. The colour variation occurs because these tracks are built on different levels, separated by thin films of insulation. A final scratch-resistant coating is applied over the entire face of the die so that only the numbered gold pads round the periphery are actually exposed. These are the electrical connections to the MMIC, some of which are used for device tests only. [Picture by kind permission of Intarsia Corporation].

\section{THE ROLE OF GOLD}

Silicon semiconductor technology makes extensive use of aluminium and copper metallizations, with aluminium preferred on the wafer and copper on the printed circuit board. Microwave devices use only gold. This metal is chosen primarily because of its high electrical conductivity and ease of processing, which stems largely from its inertness in ambient atmospheres. In order to charge a given value of circuit capacitance, the current that has to be supplied rises with increasing frequency of operation. MMICs operate at much higher frequencies than silicon integrated circuits so it is therefore necessary to provide much lower resistance DC and RF connection paths between the components. High conductivity connections also prevent excessive voltage drops and associated heating within the device. The metal must not react adversely with the semiconductor material, and whilst gold is relatively inert, thin layers of titanium and platinum are still required to provide a barrier against interaction of gold with the semiconductor material. The lustre of gold also facilitates inspection during manufacture, which is by no means easy when track widths can be below $0.2 \mu \mathrm{m}$ in places.

Gold is also to be applied extensively to the backside of MMICs. First, complex gold-based metallurgy systems are used to make an ohmic contact to the GaAs. This is then finished with a thick gold metallization to provide a low impedance ground plane and a solderable surface for mechanical attachment. Because this surface is gold, a gold-based solder such as the $80 \mathrm{wt} \%$ gold / $20 \mathrm{wt} \%$ tin eutectic alloy is invariably used to make the joint.

The circuit boards used for microwave component assembly also exploit gold for similar reasons. The substrate material in this case is a highly polished alumina tile. The gold tracks are typically $3 \mu \mathrm{m}$ thick as this provides a good compromise between electrical conductivity and precise physical definition.

The traditional method for making electrical connection between a semiconductor die and circuit board is by wire bonding. This is simply a short loop of wire that may be as little as $25 \mu \mathrm{m}$ in diameter. In cases where an extremely good contact is required between the circuit elements, gold bonding wire is often used in the place of aluminium wire, and an order of magnitude improvement in conductivity is thereby achieved.

The major growth area for gold in this technology area is interconnection between the MMIC and the substrate. Despite wire bonding being a highly reliable and versatile interconnection method, as the operating frequency increases it becomes progressively less appropriate. Although the interconnects are very short, typically only a few hundred microns in length, at microwave frequencies a bond wire functions as a large series inductor that eventually becomes so large that it cannot be accommodated by design or tuned out. Microwave devices intended for operation above about $25 \mathrm{GHz}$ exploit another form of interconnection known as flip-chip or bump bonding.

Flip-chip interconnection is a method of making simultaneous electrical connection and physical attachment between a semiconductor die and its substrate. The process involves placing a pillar of interconnection metal at each attachment point. Because the pillars can be made short and squat, very low series inductance interconnects can be realized. This is illustrated by the metrics in the following Table 1, which compares the principal interconnect characteristics of each technology: 
Table 1 Comparison of Principal Interconnect Characteristics for Wire-Bond and Flip-Chip Interconnect Technologies

$\begin{array}{lcc}\text { Characteristic Technology } & \text { Wire-Bond Interconnect } & \text { Flip-Chip Interconnect } \\ \text { Resistance, } \mathrm{mOhm} & 125 & 5 \\ \text { Inductance, } \mathrm{pH} & 2,500 & 1 \\ \text { Capacitance, fF } & 25 & 2\end{array}$

\section{FLIP-CHIP INTERCONNECTION}

Two process variants are used commercially: flip-chip solder bonding and compression bump bonding. Both rely heavily on gold or gold alloys for their function. In the first stage of the process the alumina substrate is coated with a layer of photoresist and a mask used to define the pattern of the interconnects. The apertures are then developed-out and the interconnect metal built-up by electroplating. To provide the requisite $\mathrm{RF}$ properties, the interconnect pillars are typically $30 \mu \mathrm{m}$ high by $50 \mu \mathrm{m}$ diameter.

Compression bump bonding exploits pillars of pure gold. These are aligned to mating pads on the MMIC, which of course has been inverted, and a thermal/pressure cycle applied to effect a solid state diffusion bond.

Flip-chip bonds use the $80 \mathrm{wt} \%$ gold / $20 \mathrm{wt} \%$ tin solder which melts at $280^{\circ} \mathrm{C}$. It is applied to the substrate as a two-layer deposit of tin over gold. The electroplate needs to be fused to form the eutectic alloy and this is achieved by providing a short thermal excursion above $420^{\circ} \mathrm{C}$. If this temperature is not attained then there is a risk that the constituents will not completely alloy due to the presence of the high melting point intermetallic compound, AuSn, which forms at the common interface. The MMIC is inverted and aligned, as above, and a second thermal cycle to just above the melting point of the solder provides the driving force for wetting onto the gold pads on the MMIC. The gold/tin eutectic solder is an ideal choice for this application, because a standard MMIC can then be either wire bonded or flip-chip mounted; the same gold pads being appropriate for both interconnection methods.

Flip-chip solder bonding and compression bump bonding have different characteristics. The principal difference between them is that the surface tension of the molten solder alloy, that is produced during flip-chip bonding, can be exploited to provide precise alignment between the MMIC and the substrate in all three orthogonal directions. The repeatability of manufacture that this confers has considerable benefit for the performance of microwave systems that frequently require many hundreds or even thousands of modules with identical performance to achieve the system functionality $e g$ phased array radars or communications systems.

\section{DIE ATTACH}

Despite the apparent advantages of flip-chip interconnection, many MMICs are still attached and

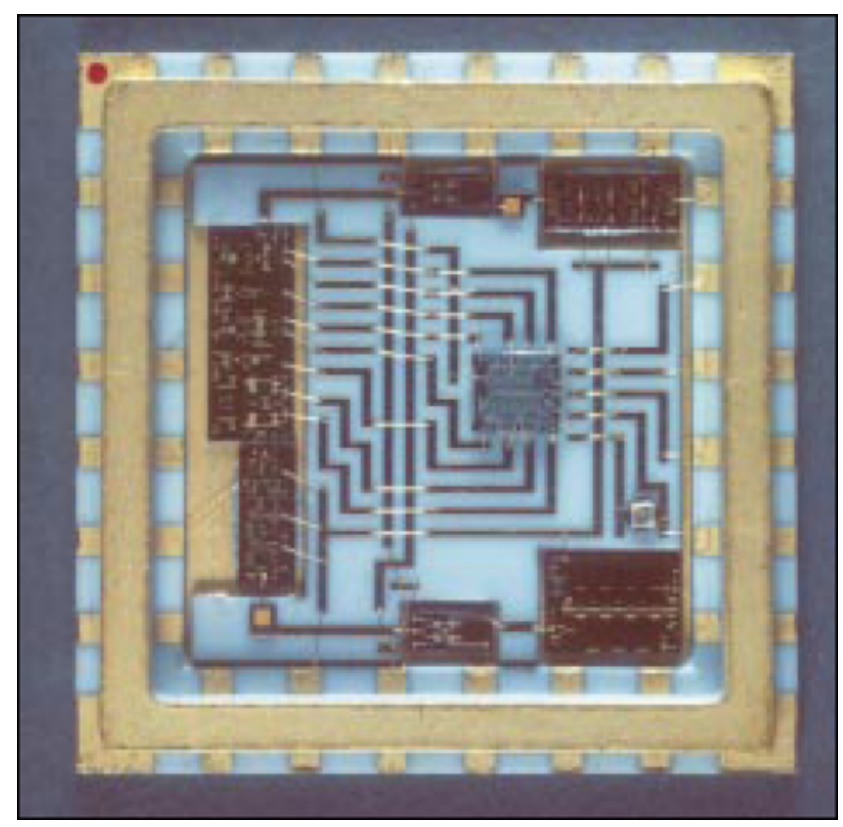

Figure 2 Wire-Bonded MMICs

Frequently a circuit will exploit more than one $M M I C$ to provide the required functionality. Here, six MMICs are arranged and interconnected in a single package together with a silicon control chip. Gold wire bonds provide the necessary connections between the MMICs, circuit board and package, although they are so fine as to be barely visible. The circuit board and package are both made of alumina. The precise definition of the tracks on the circuit board is indicative that they have been produced by a thin film process, while the package will have been built using thick-film technology. This package is designed to be hermetically sealed. This will be done by soldering a gold-plated kovar lid onto the raised seal ring, which is also gold. 
wire bonded in the traditional manner. This situation arises because semiconductors operating at microwave frequencies are highly inefficient. For example, a continuously rated GaAs transmit amplifier will have an electrical efficiency in the region of $10 \%$. Thus for each watt of radiated power, nine times that amount of heat will be generated within the chip. To protect the die against thermal breakdown it must be attached directly to a heat sink of low thermal impedance. For these MMICs that are not flip-chip bonded, the preferred method of physically attaching the goldmetallized back surface to the substrate is by fluxless soldering. The process must be fluxless because the operational life of MMICs is adversely affected if they are exposed to hydrogeneous or halide species.

Of all the solder alloys, only the gold-based eutectic compositions (namely $80 \mathrm{Au} / 20 \mathrm{Sn}, 97 \mathrm{Au} / 3 \mathrm{Si}$ and $87.5 \mathrm{Au} / 12.5 \mathrm{Ge}$; wt\%) and some of the indiumbased formulations are suitable for bonding to thick gold metallizations. The suitable indium solders are those that form a continuous intermetallic layer of the

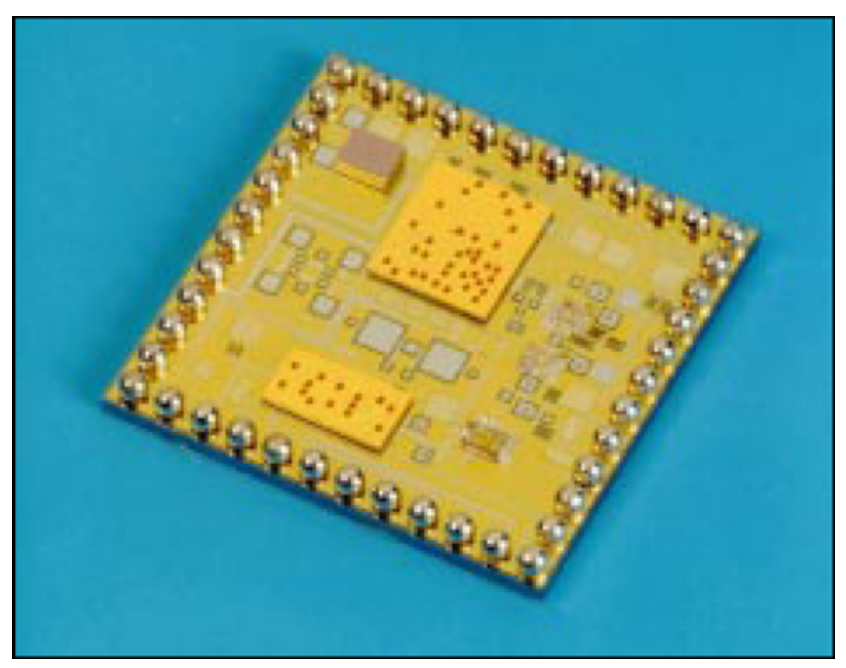

Figure 3 Flip-Chip Bonded MMICs

This picture shows an RF multi-chip module. This relatively advanced product exploits two MMICs and some silicon integrated circuits mounted on a multi-layer silicon circuit board. The MMICs are attached and interconnected by flip-chip bonding to provide the combination of low assembly cost, while maximizing $R F$ performance. The silicon devices use traditional aluminium wire bonds for their interconnects. Around the periphery of the silicon substrate is an array of large solder balls. These permit the entive assembly to itself be flip-chip mounted dinectly onto a printed circuit board, resulting in a highly miniaturized and low profile product. [Picture by kind permission of Intarsia Corporation].

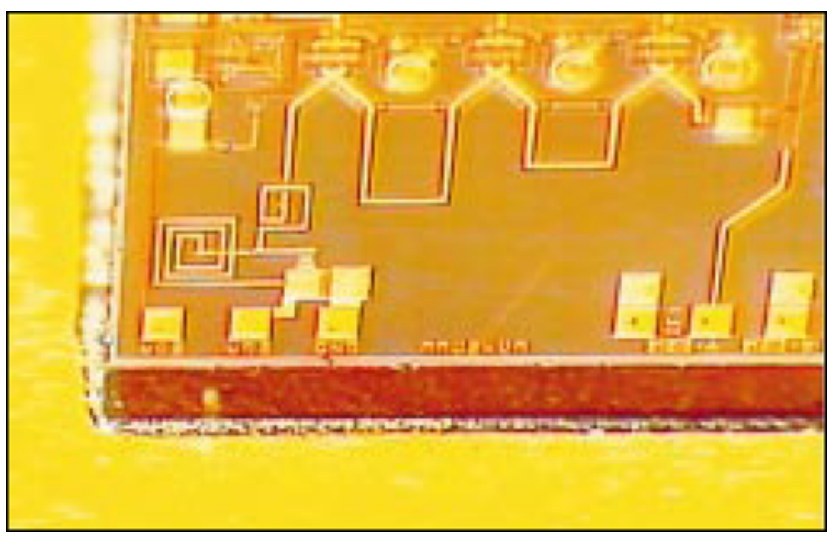

Figure 4 MMIC Die Attach

Close-up view of a soldered joint made between a MMIC (above) and a gold substrate (below). The solder is the $80 \mathrm{wt} \%$ gold $/ 20 \mathrm{wt} \%$ tin eutectic composition alloy. Despite the high proportion of gold in this solder it always appears silver in colour. This is because all of the gold present is tied up in intermetallic compounds, which are metallic white in hue. In this application it is extremely important that there an no edge opening voids. This is because the back-surface of a MMIC provides the electrical ground to the RF circuits on the front face. Any physical discontinuity in this ground path will adversely affect the device performance.

compound AuIn 2 at the common interface. Both gold and indium have very low rates of diffusion through this phase, so that once a continuous layer is established, further alloying is precluded. The three gold solders alloy with gold to the same end result but in an entirely different manner. In this case, the dissolution of gold by the molten solder abruptly raises the melting point of the advancing liquid front. The extent of solder spreading and depth of penetration into the metallization can then be effectively regulated by the process temperature.

Indium-based solders tend not to be used for two reasons. First, they have melting points mostly below $220^{\circ} \mathrm{C}$, which precludes subsequent assembly operations using standard lead-tin solder. Second, indium solders are difficult to employ without flux. The problem is the native oxide film that forms during storage in air and without flux there is no means of removing this barrier to wetting and spreading. The gold-rich solders are inherently more noble in character and hence are much simpler to exploit for this application. Their melting points are also comfortably higher than the process temperature for lead-tin solder, thereby facilitating step soldering operations. 


\section{CONCLUSIONS}

Microwave electronics is one of the fastest growing industrial sectors. Fuelled by the need for an even greater number of communications networks of increasing bandwidth, the compound annual growth rate for microwave systems is predicted to increase from the already challenging rate of $14 \%$ to $20 \%$ by 2002. Gold is used exclusively for the tracking and interconnects. Its pre-eminent position in providing this function is due to its combination of high electrical conductivity, easy compatibility with semiconductor processing and versatility in the number and range of joining and interconnection techniques to which it is suited.

\section{ACKNOWLEDGEMENTS}

The assistance provided by D M Brookbanks and S D Wadsworth in the writing of this article and supply of the illustrations is gratefully acknowledged.

\section{ABOUT THE AUTHOR}

Dr Giles Humpston was awarded a first degree in Metallurgy from Brunel University in 1983, followed by a $\mathrm{PhD}$ on the constitution of solder alloys 1985 . He has since been employed by GEC and now leads the Future Technologies Division of Marconi Materials Technology, a role that not only includes responsibility for projects involving electronic and optical devices but extends to magnetic materials, electroceramics, diamond and fuel cells. He is the cited inventor on 15 patents and the author of over 50 papers. $\mathrm{He}$ is coauthor of the book 'Principles of Soldering and Brazing, published by ASM International in 1993. He is the recipient of six international awards, the most recent of which include the Cook Prize of the Institute of Materials and the Robert L Peaslee Brazing Award of the American Welding Society.

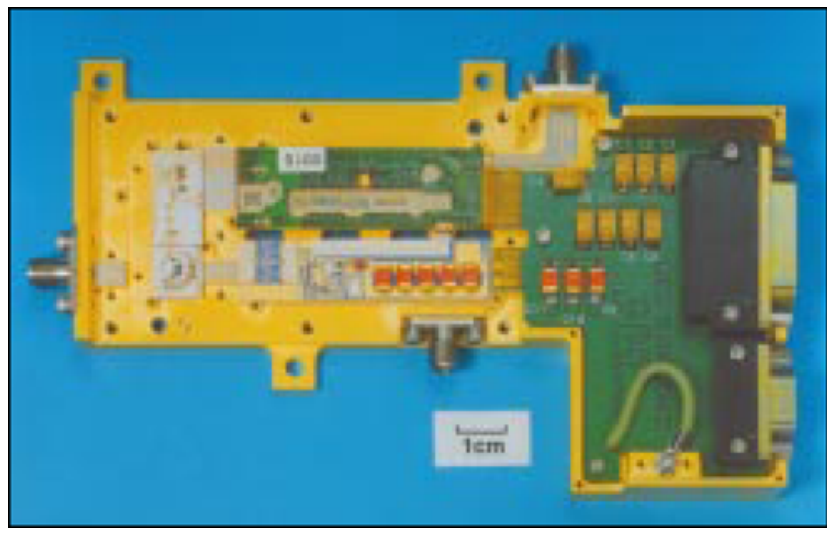

Figure 5 High Specification RF Module

The RF module shown exploits a diversity of materials to deliver the required functions. Gold is an essential element in virtually every aspect of its construction. The large green printed circuit board to the right supports a number of large capacitors and two control connectors. It is made from a special type of FR4 with buried gold tracks. Three of the capacitors have gold terminations. The smaller green board is PTFE, also with gold tracks. In a cavity down the centre of this board can be seen the functional MMICs. There are 14 MMICs in total. These are attached using gold solder and interconnected by gold wire bonds. The blue circuit boards are built using thick film technology and support large areas of gold metallization. The white circuit boards are alumina metallized with thin film-gold. Although not visible in the picture, the connector pins are gold plated to ensure low resistance and reliable connections to the mating plugs. Finally, the housing is an aluminium alloy that has been coated with nickel, overlaid with 3-5 $\mu \mathrm{m}$ of gold. This is an areospace standard finish, proven to provide a corrosion resistant coating of high electrical conductivity. 